\title{
Temporomandibular disorder modifies cortical response to tactile stimulation
}

\author{
Mary Beth Nebel ${ }^{1,2}$, Stephen Folger ${ }^{4}$, Mark Tommerdahl ${ }^{2}$, Mark Hollins ${ }^{3}$, Francis \\ McGlone $^{5}$, and Gregory Essick ${ }^{1}$ \\ ${ }^{1}$ Center for Neurosensory Disorders, University of North Carolina, Chapel Hill, NC \\ ${ }^{2}$ Department of Biomedical Engineering, University of North Carolina, Chapel Hill, NC \\ ${ }^{3}$ Department of Psychology, University of North Carolina, Chapel Hill, NC \\ ${ }^{4}$ Department of Physical Therapy Education, Elon University, Elon, NC \\ ${ }^{5}$ Department of Neurological Sciences, University of Liverpool, Liverpool, UK
}

\begin{abstract}
Individuals with temporomandibular disorder (TMD) suffer from persistent facial pain and exhibit abnormal sensitivity to tactile stimulation. To better understand the pathophysiological mechanisms underlying TMD, we investigated cortical correlates of this abnormal sensitivity to touch. Using functional magnetic resonance imaging (fMRI), we recorded cortical responses evoked by low frequency vibration of the index finger in subjects with TMD and in healthy controls (HC). Distinct subregions of contralateral SI, SII, and insular cortex responded maximally for each group. Although the stimulus was inaudible, primary auditory cortex was activated in TMDs. TMDs also showed greater activation bilaterally in anterior cingulate cortex and contralaterally in the amygdala. Differences between TMDs and HCs in responses evoked by innocuous vibrotactile stimulation within SI, SII, and the insula paralleled previously reported differences in responses evoked by noxious and innocuous stimulation, respectively, in healthy individuals. This unexpected result may reflect a disruption of the normal balance between central resources dedicated to processing innocuous and noxious input, manifesting itself as increased readiness of the pain matrix for activation by even innocuous input. Activation of the amygdala in our TMD group could reflect the establishment of aversive associations with tactile stimulation due to the persistence of pain.
\end{abstract}

Perspective-This article presents evidence that central processing of innocuous tactile stimulation is abnormal in TMD. Understanding the complexity of sensory disruption in chronic pain could lead to improved methods for assessing cerebral cortical function in these patients.

\section{Keywords}

Temporomandibular disorders (TMD); fMRI; cortical imbalance; SI; chronic pain

(C) 2010 The American Pain Society. Published by Elsevier Inc. All rights reserved.

Corresponding author: Gregory Essick 2112 Old Dental Building CB \#7455 University of North Carolina Chapel Hill, NC 27599-7455 Telephone: (919) 966-5680 Fax: (919) 966-5339 greg_essick@ dentistry.unc.edu.

Publisher's Disclaimer: This is a PDF file of an unedited manuscript that has been accepted for publication. As a service to our customers we are providing this early version of the manuscript. The manuscript will undergo copyediting, typesetting, and review of the resulting proof before it is published in its final citable form. Please note that during the production process errors may be discovered which could affect the content, and all legal disclaimers that apply to the journal pertain. 


\section{Introduction}

A considerable body of evidence suggests that painful conditions are often accompanied by alterations in cutaneous sensory perception. Nathan reported that localized pain due to peripheral or central lesions can impair the perception of tactile stimuli within the painful region 51, similarly, provoking pain in patients with pathological pain (e.g., tennis elbow) increases tactile detection thresholds in the area of pain referral ${ }^{44}$. In some clinical conditions, widespread impairment of tactile sensitivity has been documented. Patients with chronic cervicobrachialgia 70 and persistent patellofemoral pain 38 demonstrate systemic elevation of vibrotactile detection thresholds compared to healthy controls. Although the clinical presentations of these conditions differ, there is increasing recognition that systematic assessment of somatosensory perception in disorders characterized by persistent pain would greatly aid diagnosis and evaluation of treatment efficacy.

One condition in which local and widespread sensory disturbances have been examined is temporomandibular disorder (TMD), a non-specific diagnosis representing a constellation of conditions characterized by persistent facial pain and impaired oral function 14. TMD, the most common chronic orofacial pain condition in the United States, impacts approximately $12 \%$ of the population 13. Individuals with TMD frequently report pain in widespread body areas 30,75 , suggesting that central pathophysiological processes contribute to the persistence of pain. In addition, TMD is associated with several co-morbid functional syndromes including fibromyalgia (18\%) 57, vulvar vestibulitis 78, and irritable bowel syndrome (64\%) 1 .

Vibrotactile sensibility on the face of TMD patients is characterized by elevated detection threshold ${ }^{34}$ and impaired frequency discrimination ${ }^{33}$, a process shown to rely on intact somatosensory cortex ${ }^{42}$. Outside of the painful region, a marginal increase in vibrotactile detection threshold ${ }^{33}$ is overshadowed by perceptual amplification of the intensity of suprathreshold tactile stimuli ${ }^{32}$.

One interpretation of the association between persistent pain and abnormal tactile sensibility is that there is a disturbance in the normal balance between cortical noxious and non-noxious processing. Animal studies and neural network modeling indicate that regions of somatosensory cortex dominated by input from different spinal pathways interact disadvantageously when normal input is disrupted, for instance, by dorsal column transection

68. Tissue injury and inflammation have also been shown to alter cortical responsivity to noxious and non-noxious stimulation in animal models of arthritis ${ }^{29}, 43$. In addition, neuroimaging studies of phantom limb pain reveal a correlation between cortical reorganization of somatic processing and the magnitude of pain experienced ${ }^{5,}{ }^{20}$; however, pain coexists with extensive sensorimotor deafferentation which also contributes to cortical reorganization. Whether the vibrotactile perception impairments observed in individuals with TMD pain likewise reflect an abnormal topography of cortical somatosensory processing remains to be determined.

The purpose of the present study was to determine, using functional magnetic resonance imaging (fMRI), whether the decreased sensitivity to touch observed in TMD is associated with alterations in the magnitude and location of brain activity evoked by low frequency skin vibration.

\section{Materials and Methods}

\section{Subjects}

Twenty-five women consented to a protocol approved by the Institutional Review Board at UNC-Chapel Hill Medical Center. The sample population was restricted to women because 
the prevalence of TMD is significantly higher in women; 2 to 1 in the general population and 8 to 1 in the clinical setting 8. Thirteen participants fulfilled Research Diagnostic Criteria (RDC) for TMD ${ }^{14}$, average age (SD) was 28.7 (7.6) years; the other twelve participants were neurologically healthy controls whose average age was 28.8 (7.9) years. Immediately prior to the imaging session, each participant completed the Short-form McGill Pain Questionnaire (SF-MPQ) to assess her current level of pain 48.

\section{Stimulation}

While in the MRI scanner, low frequency vibration (tactile flutter) was applied to the distal pad of the right index finger using a purpose-designed piezoelectric tactile stimulator (PTS) 22. Tactile stimuli were applied to the hand rather than to the temporomandibular region to identify the presence of global abnormalities in central somatosensory processing that could not be attributed to abnormalities in stimulus-evoked afferent activity from the site of the patients' pain complaints. A static surround limited the stimulation to a region under the $8-\mathrm{mm}$ diameter Teflon contactor, which was attached to the bender element. Consistent with previous neuroimaging investigations of somatosensory cortex in primates, a $26 \mathrm{~Hz}$ sinusoidal stimulus with peak-to-peak amplitude of $400 \mu \mathrm{m}$ was used. Flutter stimulation near this frequency generates robust and repeatable optical intrinsic signal (OIS) responses within the post-central gyrus in squirrel monkeys 62 . Flutter stimulus events were $4 \mathrm{~s}$ in duration and repeated every 32 s to allow adequate observation of the hemodynamic response to each event. Subjects were instructed to keep their eyes closed and to focus attention on the presence of the stimulus. Twenty-three of the 25 subjects participated in two imaging sessions during which two functional imaging series of tactile flutter were completed. Each imaging series consisted of 14 flutter stimulus presentations for a total of 56 events. Two subjects (one TMD) completed a single imaging session for a total of 28 events. At the end of each imaging series, subjects were asked to rate the average intensity of the flutter stimulus using a labeled magnitude scale with the following anchor points: felt nothing (0), barely detectable(1.5), weak vibration(5), moderate vibration(16), strong vibration (33), very strong(50), and most intense vibration imaginable (100). Subjects were instructed to first choose the most appropriate label range to describe the intensity of the stimulus and then convert that into a number. Subjects were familiarized with the scale and presented with two test stimuli to rate before entering the scanner room.

\section{Imaging Parameters}

Scanning was performed on a Siemens Magnetom Allegra, head-dedicated 3.0T scanner system with $40-\mathrm{mT} / \mathrm{m}$ gradients and a $30 \mathrm{~cm}$ radio frequency (RF) volume coil. Subject head motion was restricted using foam cushions, and earplugs and earphones were worn by subjects to reduce scanner noise. A total of 160 contiguous, high-resolution images covering the entire brain were acquired using a magnetization prepared rapid gradient echo (MPRAGE) T1weighted sequence (TR: $1700 \mathrm{~ms}$, Echo Time (TE): $4.38 \mathrm{~ms}$, Flip angle: $8,1 \mathrm{~mm}$ isotropic sampling). These structural images were aligned near-axially, parallel to the plane underlying the rostrum and splenium of the corpus callosum and were used for coregistration with the functional data. Whole brain functional images consisted of 50 slices collected using a gradient echo pulse sequence sensitive to blood oxygenation level dependent (BOLD) contrast with echo planar k-space sampling at a repetition rate (TR) of 3000ms (TE: 30ms, Flip angle: 90, Image matrix: $64 \times 64$, isotropic voxel size: $3 \mathrm{~mm}^{3}$ ). The functional images were aligned similarly to the structural images. A semi-automated, high-order shimming program ensured global field homogeneity. Imaging series began with two discarded RF excitations to allow the change in net magnetization of the sample following excitation to reach steady state equilibrium. 


\section{Image Data Analysis}

Before any statistical analyses were performed, the following preprocessing steps were applied to the fMRI data to remove task-independent variability using FMRIB Software Library (FSL) version 4.1.2 64,74: (i) brain extraction for non-brain removal 63, (ii) subject motion correction using MCFLIRT 36, (iii) temporal realignment to adjust for slice acquisition order using Fourier-space time-series phase shifting, (iv) spatial smoothing using a Gaussian filter with a FWHM 5mm kernel to boost the signal to noise ratio of the data, (v) grand-mean intensity scaling of the entire 4D dataset by a single factor, and (vi) high-pass temporal filtering to remove low frequency artifacts. Functional images of each subject were co-registered to structural images in native space, and structural images were warped into Montreal Neurological Institute (MNI) stereotaxic space to allow for intersubject comparison. The same transformation matrices used for structural-to-standard transformations were then applied to the co-registered functional images, and all registrations were carried out using an intermodal registration tool (affine, 12 degrees of freedom). Voxel-wise temporal autocorrelation was estimated and corrected using FMRIB's Improved Linear Model 37.

Onset times of tactile flutter events were used to generate a regressor to model the hemodynamic response (HDR) to the stimulus. Model fitting generated whole brain images of parameter estimates and variances, representing average signal change from baseline. Groupwise activation images were calculated by a mixed effects higher level analysis using Bayesian estimation techniques, FMRIB Local Analysis of Mixed Effects, with a cluster mean threshold of $\mathrm{z}>2.5$ and a cluster corrected significance of $\mathrm{p}<0.054$. Following statistical thresholding, mixed effects group contrast images were restricted to voxels in which a significant, cluster corrected HDR was evoked by skin flutter in either group composing the contrast. The Jülich histologic atlas 15,17 and the Harvard-Oxford cortical and subcortical structural atlases (Harvard Center for Morphometric Analysis, Charlestown, MA) were used to localize activation clusters 16,18 . The final fMRI analysis step consisted of extracting average BOLD time courses from functional regions of interest (ROIs) identified to differentiate groups based on whole-brain analyses described above. Peak responses were compared between groups in these regions.

\section{Results}

\section{Self-reported Present Pain}

On average, TMD subjects reported their present pain intensity on the day of testing to be 2.4 on a $10 \mathrm{~cm}$ visual analog scale with end labels of no pain (0) and worst possible pain (10). Control subjects reported an average present pain intensity of 0.16 out of 10 on the day of testing.

\section{Perceptual Ratings}

On average, the TMD group rated the intensity of the flutter stimulation as $32.0(\mathrm{SD}=15.4)$, corresponding to a level of "strong" on the labeled magnitude scale while the control group rated the intensity of the same stimuli as only 19.2 , on average $(\mathrm{SD}=12.5)$, corresponding to moderately intense. A t-test indicated that this difference in mean perceived intensity was significant $(\mathrm{p}=0.03)$.

\section{Imaging Data}

Individual Group Analysis-In a repeated measures analysis, no significant differences in the response to tactile flutter were observed between imaging sessions for either group; accordingly, for each subject who completed two sessions, data from the two sessions were combined in subsequent analyses. For both groups, skin flutter evoked significant 
hemodynamic responses in established somatosensory processing areas, namely contralateral primary somatosensory cortex (SI), bilateral secondary somatosensory cortex (SII) and bilateral insular cortex. In addition, robust responses were evoked in both groups in sensory association areas, bilateral anterior cingulate cortex (ACC) and ipsilateral inferior parietal lobule, as well as in ipsilateral middle frontal gyrus, an area associated with attention to transient targets. Figure 1 illustrates the pattern of activation for each group in these regions, and Table 1 indicates the MNI coordinates of all significant activation clusters in the control group while Table 2 lists the coordinates of all significant activation clusters in the TMD group. Up to four local maxima within each activation cluster are listed since several clusters span more than one cortical region.

Between-group analyses-Different patterns of activation in response to skin flutter were observed for the TMD and control groups. Direct comparison of (control - TMD) and (TMD - control) flutter contrasts revealed areas within the above mentioned clusters in which one group demonstrated significantly greater activation than the other; Table 3 lists MNI coordinates of all active regions demonstrating a significant group effect.

SI-Both the control group and the TMD group displayed significant responses in contralateral SI and SII; however, Figure 2 illustrates the distinct centers of activation within these regions for the two groups. The SI center of activation for the control group ("A" in Figure 2) was posterior and lateral to the SI center of activation for the TMD group ("B" in Figure 2) according to the MNI coordinates listed in Table 3. Figure 2 also indicates average hemodynamic time courses for both groups derived from contralateral SI voxels identified by the whole brain analysis to differentiate between groups. In the posterior region of area 1, the response of the control group was significantly greater than that of the TMD group 3-9 seconds after the onset of skin flutter stimulation ( $\mathrm{p}<0.01$ for all three time points); see " $A$ " in Figure 2. In the more anterior portion of SI, the peak of the TMD HDR at 3 seconds was significantly greater than that of the controls $(\mathrm{p}<0.04)$; see "B" in Figure 2.

SII and primary auditory cortex (A1) - Separation between groups also occurred in contralateral SII, with the mass of the control HDR ("C" in Figure 2) residing in parietal operculum subregions OP1 and OP4 ${ }^{15},{ }^{17}$ and with the TMD group's SII activation extending from OP1 ("D" in Figure 2) across the Sylvian fissure and into neighboring primary auditory cortex ("E" in Figure 2). Local maxima were identified on either side of the Sylvian fissure in both the TMD activation maps (Table 2) and the TMD - control contrast maps (Table 3). Using the Jülich histologic atlas, it was determined that $20 \%$ of the contralateral SII cluster listed in Table 3 resided in primary auditory cortex (A1) ${ }^{49}$. No statistical difference was observed between the time to or magnitude of peak TMD HDR in SII and A1. On the ipsilateral side, no region of SII demonstrated greater activation to skin flutter in the control group than in the TMD group. The TMD group showed greater activation than the control group in OP1 and again this activation extended into primary auditory cortex (Figure 3); approximately $24 \%$ of the cluster labeled ipsilateral SII in Table 3 was located in ipsilateral A1.

SII and A1 are located adjacently on opposite banks of the Sylvian fissure, and previous research has suggested that extensive overlap may occur in fMRI responses evoked by tactile and auditory stimulation when data is combined across subjects due to their close anatomical proximity ${ }^{54}$. To verify that activation of primary auditory cortex was not caused by a misregistration of individual subject data onto the standard atlas, we inspected subject responses on their individual high-resolution anatomical images. Activation of contralateral A1 was found in all 13 TMD subjects and activation of ipsilateral A1 was found in 9 of 13 TMD subjects. Figure 4 contains fMRI activations evoked by tactile stimulation from two exemplary TMD subjects and one healthy control; activation clearly extends into A1 for both TMD subjects but remains in SII for the control subject. 
Insula, ACC \& amgydala-Figure 5 depicts brain areas outside of those regions traditionally associated with tactile processing in which the TMD group showed greater activation than controls. Although both groups displayed bilateral ACC activation, the control group's ACC HDR was surpassed in magnitude and spatial extent by the HDR of the TMD group; see "A" in Figure 5. The between-group flutter contrast also revealed a dissociation of the HDR in contralateral insular cortex. The control group demonstrated greater evoked activity in an anterior region of the insula while conversely, the TMD group showed greater evoked activity in a more posterior region ("B" in Figure 5). Unexpectedly, activation evoked by skin flutter was also greater for the TMD group in the contralateral amygdala; refer to " $\mathrm{C}$ " in Figure 5 .

\section{Discussion}

To the best of our knowledge, the present report is the only examination of brain activity evoked by innocuous vibrotactile digit stimulation in TMD patients. The gross morphology of cortical activation elicited by skin flutter in our controls was similar to patterns previously reported $22,40,45,47$, including contiguous activation of SI and $\mathrm{SII}^{6}$. The results are also consistent with the hypothesis that, in TMD, differences exist in the location and magnitude of cortical processing of vibrotactile stimulation, adding more evidence to our understanding of the disruption of the somatosensory system in a chronic pain condition.

The finding that, on average, TMD subjects perceived flutter stimulation as more intense than controls is consistent with published reports of TMD patients experiencing perceptual amplification of innocuous levels of pressure stimulation, rating weak pressures as more intense compared to controls ${ }^{32}$. These ratings should be interpreted with caution since we did not perform a rigorous calibration of the scale with each participant to minimize intersubject differences in its use.

\section{SI}

Group comparisons revealed differences between controls and TMDs in evoked activity within the hand region of SI. The SI hand region is composed of a number of cytoarchitectonically defined subdivisions (areas 3a, 3b, 1, and 2) and the Jülich histologic atlas indicated that the SI cluster in which the control group demonstrated greater activation than the TMD group belonged to area 1, while the SI cluster in which the TMD group showed greater activation than controls was more anterior and medial (Table 3), and spanned areas 1 and $3 b^{26},{ }^{27}$. Attempting to assign cytoarchitectonic labels to fMRI activation foci is prone to error due to substantial image processing and inter-subject variability ${ }^{27}$; however, areas $3 \mathrm{~b}$ and 1 are traditionally regarded as the SI core for processing input from cutaneous receptors. The stability of the location and spatial extent of SI activity evoked by flutter of increasing intensity has been demonstrated by $\mathrm{OIS}^{62}$ and fMRI monkey studies ${ }^{77}$, suggesting that this group difference in the location of maximal response is not simply reflective of greater tactile intensity experienced by TMDs. Painful and innocuous stimuli appear to drive different neuronal populations within somatosensory cortex, and the general orientation of the shift in maximal BOLD response between controls and TMDs is similar to the pattern of response observed when comparing activity evoked by innocuous and noxious stimulation of the hand in both healthy humans ${ }^{50,53,65}$ and in squirrel monkeys ${ }^{66,67,72}$; the fringe of SI that responds to painful stimulation of the hand is anterior and medial to the core SI hand tactile locus.

Changes in SI tactile responsivity have been studied in patients with other persistently painful conditions with mixed results. Using fMRI to study complex regional pain syndrome (CRPS), Pleger et al observed a reduction in SI activity evoked by tactile stimulation in CRPS compared to healthy controls ${ }^{56}$ while the CRPS subjects in the magnetoencephalography (MEG) study of Vartiainen et al demonstrated enhanced SI responsivity to tactile stimulation compared to 
controls 69. Accounting for methodological differences, we consider both of these results consistent with our findings. The SI subregion in which our chronic pain group showed decreased activity compared to controls was located near the crown of the postcentral gyrus, making it difficult to detect using MEG which is intrinsically insensitive to radially oriented flow. A weaker magnet in the Pleger study necessitated the use of larger voxels and increased spatial smoothing; partial volume effects could have caused blurring of activity within the two distinct SI subregions we identified to show opposing group effects, with the net effect being decreased evoked activity in the chronic pain state.

\section{SII \& A1}

The group differences we observed in the SII response to flutter appear to be consistent with comparisons of SII responsiveness to innocuous versus noxious stimulation in healthy subjects. The contralateral SII locus of activation for the control group was anterior to the SII locus of activation for the TMD group. One of the earliest monkey electrophysiological studies suggested that anterior SII consisted of neurons responsive to tactile input while posterior SII included polysensory and nociceptive neurons ${ }^{73}$. In a more recent meta-analysis of reported SII activations from human functional imaging studies of hand stimulation, Eickhoff et al found that SII voxels associated with non-painful stimulation, situated at the border between cytoarchitectonically defined OP1 and OP4, were anterior to SII voxels in OP1 associated with pain-related activity ${ }^{15}$. Additionally, Ferretti et al demonstrated two distinct SII subregions of activation in the anterior-posterior direction, with only the posterior subregion of activation exhibiting modulation due to pain intensity ${ }^{19}$. The activation of the posterior subregion of SII by innocuous stimulation in our TMD group further suggests that this stimulation engaged circuits normally reserved for processing noxious stimulation.

Both groups exhibited a BOLD response in ipsilateral SII. However, the response of the TMD group was greater in magnitude and spatial extent. Pain-related activity has been shown to be more widely dispersed on both sides of the cortex than activity evoked by innocuous vibrotactile stimulation in pain-free subjects, ${ }^{11},{ }^{12}$ and rat models of neuropathic pain have demonstrated bilateral increases in somatosensory cortex responsivity ${ }^{46}$. Thus, the recruitment of additional SII processing resources on the ipsilateral side in TMD further implicates an influence of TMD pain on the processing of the vibrotactile stimuli.

Given that many activities that produce tactile sensations also produce sound, it is not surprising that a growing body of evidence suggests that tactile stimulation can activate auditory cortex $9,21,23,24,60$ and that horizontal connections between auditory cortex and somatosensory cortex exist ${ }^{7}, 10,23$. This close anatomical and physiological relationship between cortical regions nominally belonging to separate modalities may help to explain behavioral interactions between hearing and touch $28,39,76$. What is surprising is that our TMD group, using conservative spatial smoothing ${ }^{60}$, showed greater activation in primary auditory cortex than our control group. The results suggest that the posterior subregion of SII (activated in our TMD group) has readier access to A1, by reason of anatomical proximity, than does the anterior subregion of SII (activated in our HC group), raising the intriguing possibility that behavioral interactions between somatosensation and hearing might be more substantial in TMD patients than in controls and that auditory responses to some stimuli occur even when they are inaudible to the ear. Indeed, somatosensory input can modulate the intensity and character of tinnitus 55 , the symptoms of which are more common in individuals with TMD than in the general population 25 . Further investigation of the connectivity between somatosensory and auditory cortex in the human brain is needed before any definite conclusions can be drawn. 


\section{Insula, ACC \& amgydala}

Also surprising was that flutter stimulation evoked activity in the contralateral amygdala of our TMD group. To our knowledge, no neuroimaging investigation of innocuous tactile stimulation in humans has demonstrated a significant response in the amygdala; however, animal studies have provided evidence of amygdala sensitization following the induction of an inflammatory chronic pain state ${ }^{52}$ and have emphasized the role of the amygdala ${ }^{31,52}$ as well as the insula ${ }^{35}$ and $\mathrm{ACC}^{61}$ in the modulation of pain behavior, all three of which showed greater activation in our TMD group than in our control group. The amygdala plays a critical role in learning the association between aversive and neutral stimuli in classical conditioning ${ }^{41}$, and amygdala activation in response to what should be an affectively neutral stimulus could be consistent with the hypothesis proposed by Apkarian that chronic pain is a state of continuous learning in which aversive associations are continuously made with incidental events, like innocuous tactile stimulation, due to the persistent presence of pain ${ }^{3}$. Drawing conclusions about the emotional implications of amygdala activation is beyond the scope of this study, and given the association between TMD and hypervigilance ${ }^{58}$, we must also recognize the possible influence of attentional differences on processing in the insula ${ }^{2}$ and $\mathrm{ACC}^{71}$. However, the expectation of pain has been shown to increase the BOLD response evoked by nonpainful stimulation in the insula and $\mathrm{ACC}^{59}$, and similar to the dissociation of group activations we observed within SI and SII, the subregion of the insula in which our TMD group showed maximal activity reportedly responds to noxious but not to innocuous stimuli 50 .

A limiting factor of the present study is our sample size; although the number of subjects included in this study is comparable to many functional neuroimaging investigations, it may be small considering the heterogeneity in the clinical presentation of TMD. Despite this heterogeneity, we detected a disruption in the cortical processing of innocuous vibrotactile digit stimulation in TMD, and considered together, these subtle, yet significant differences suggest cortical plasticity in TMD which primes areas to respond to innocuous vibrotactile input that normally would not, including parts of the pain matrix and auditory cortex. Further investigation of how these processing differences are influenced by concurrent acute pain could help to explain their functional significance. Improving our understanding of the complexity of sensory disruption in chronic pain could allow for the development of more accurate chronic pain models needed to test and improve the efficacy of therapeutic interventions.

\section{Acknowledgments}

The authors would like to thank Steven Smith and MRI technologists Kathy Wilber, Amber Abernethy, James Barnwell, and Emilie Kearns for assistance with data acquisition as well as Ollie Monbureau and Mike Young for technical assistance. This work was supported by NIH grant P01 NS045685.

\section{References}

1. Aaron LA, Burke MM, Buchwald D. Overlapping conditions among patients with chronic fatigue syndrome, fibromyalgia, and temporomandibular disorder. Arch Intern Med 2000;160:221-7. [PubMed: 10647761]

2. Albanese MC, Duerden EG, Bohotin V, Rainville P, Duncan GH. Differential effects of cognitive demand on human cortical activation associated with vibrotactile stimulation. J Neurophysiol 2009;102:1623-31. [PubMed: 19553476]

3. Apkarian AV. Pain perception in relation to emotional learning. Curr Opin Neurobiol 2008;18:4648. [PubMed: 18835354]

4. Beckmann CF, Jenkinson M, Smith SM. General multilevel linear modeling for group analysis in fmri. Neuroimage 2003;20:1052-63. [PubMed: 14568475] 
5. Birbaumer N, Lutzenberger W, Montoya P, Larbig W, Unertl K, Topfner S, Grodd W, Taub E, Flor $\mathrm{H}$. Effects of regional anesthesia on phantom limb pain are mirrored in changes in cortical reorganization. J Neurosci 1997;17:5503-8. [PubMed: 9204932]

6. Blatow M, Nennig E, Sarpaczki E, Reinhardt J, Schlieter M, Herweh C, Rasche D, Tronnier VM, Sartor $\mathrm{K}$, Stippich C. Altered somatosensory processing in trigeminal neuralgia. Hum Brain Mapp 2009;30:3495-508. [PubMed: 19365802]

7. Budinger E, Scheich H. Anatomical connections suitable for the direct processing of neuronal information of different modalities via the rodent primary auditory cortex. Hear Res. 2009

8. Bush FM, Harkins SW, Harrington WG, Price DD. Analysis of gender effects on pain perception and symptom presentation in temporomandibular pain. Pain 1993;53:73-80. [PubMed: 8316393]

9. Caetano G, Jousmaki V. Evidence of vibrotactile input to human auditory cortex. Neuroimage 2006;29:15-28. [PubMed: 16168673]

10. Cappe C, Barone P. Heteromodal connections supporting multisensory integration at low levels of cortical processing in the monkey. Eur J Neurosci 2005;22:2886-902. [PubMed: 16324124]

11. Coghill RC, Sang CN, Maisog JM, Iadarola MJ. Pain intensity processing within the human brain: A bilateral, distributed mechanism. J Neurophysiol 1999;82:1934-43. [PubMed: 10515983]

12. Coghill RC, Talbot JD, Evans AC, Meyer E, Gjedde A, Bushnell MC, Duncan GH. Distributed processing of pain and vibration by the human brain. J Neurosci 1994;14:4095-108. [PubMed: 8027764]

13. Dworkin SF, Huggins KH, LeResche L, Von Korff M, Howard J, Truelove E, Sommers E. Epidemiology of signs and symptoms in temporomandibular disorders: Clinical signs in cases and controls. J Am Dent Assoc 1990;120:273-81. [PubMed: 2312947]

14. Dworkin SF, LeResche L. Research diagnostic criteria for temporomandibular disorders: Review, criteria, examinations and specifications, critique. J Craniomandib Disord 1992;6:301-55. [PubMed: 1298767]

15. Eickhoff SB, Amunts K, Mohlberg H, Zilles K. The human parietal operculum. Ii. Stereotaxic maps and correlation with functional imaging results. Cereb Cortex 2006;16:268-79. [PubMed: 15888606]

16. Eickhoff SB, Paus T, Caspers S, Grosbras MH, Evans AC, Zilles K, Amunts K. Assignment of functional activations to probabilistic cytoarchitectonic areas revisited. Neuroimage 2007;36:51121. [PubMed: 17499520]

17. Eickhoff SB, Schleicher A, Zilles K, Amunts K. The human parietal operculum. I. Cytoarchitectonic mapping of subdivisions. Cereb Cortex 2006;16:254-67. [PubMed: 15888607]

18. Eickhoff SB, Stephan KE, Mohlberg H, Grefkes C, Fink GR, Amunts K, Zilles K. A new spm toolbox for combining probabilistic cytoarchitectonic maps and functional imaging data. Neuroimage 2005;25:1325-35. [PubMed: 15850749]

19. Ferretti A, Babiloni C, Gratta CD, Caulo M, Tartaro A, Bonomo L, Rossini PM, Romani GL. Functional topography of the secondary somatosensory cortex for nonpainful and painful stimuli: An fmri study. Neuroimage 2003;20:1625-38. [PubMed: 14642473]

20. Flor H, Elbert T, Knecht S, Wienbruch C, Pantev C, Birbaumer N, Larbig W, Taub E. Phantom-limb pain as a perceptual correlate of cortical reorganization following arm amputation. Nature 1995;375:482-4. [PubMed: 7777055]

21. Foxe JJ, Wylie GR, Martinez A, Schroeder CE, Javitt DC, Guilfoyle D, Ritter W, Murray MM. Auditory-somatosensory multisensory processing in auditory association cortex: An fmri study. $\mathbf{J}$ Neurophysiol 2002;88:540-3. [PubMed: 12091578]

22. Francis ST, Kelly EF, Bowtell R, Dunseath WJ, Folger SE, McGlone F. Fmri of the responses to vibratory stimulation of digit tips. Neuroimage 2000;11:188-202. [PubMed: 10694461]

23. Frostig RD, Xiong Y, Chen-Bee CH, Kvasnak E, Stehberg J. Large-scale organization of rat sensorimotor cortex based on a motif of large activation spreads. J Neurosci 2008;28:13274-84. [PubMed: 19052219]

24. Fu KM, Johnston TA, Shah AS, Arnold L, Smiley J, Hackett TA, Garraghty PE, Schroeder CE. Auditory cortical neurons respond to somatosensory stimulation. J Neurosci 2003;23:7510-5. [PubMed: 12930789]

25. Gelb H, Gelb ML, Wagner ML. The relationship of tinnitus to craniocervical mandibular disorders. Cranio 1997;15:136-43. [PubMed: 9586516] 
26. Geyer S, Schleicher A, Zilles K. Areas 3a, 3b, and 1 of human primary somatosensory cortex. Neuroimage 1999;10:63-83. [PubMed: 10385582]

27. Geyer S, Schormann T, Mohlberg H, Zilles K. Areas 3a, 3b, and 1 of human primary somatosensory cortex. Part 2. Spatial normalization to standard anatomical space. Neuroimage 2000;11:684-96. [PubMed: 10860796]

28. Guest S, Catmur C, Lloyd D, Spence C. Audiotactile interactions in roughness perception. Exp Brain Res 2002;146:161-71. [PubMed: 12195518]

29. Guilbaud G, Peschanski M, Briand A, Gautron M. The organization of spinal pathways to ventrobasal thalamus in an experimental model of pain (the arthritic rat). An electrophysiological study. Pain 1986;26:301-12. [PubMed: 3774350]

30. Hagberg C. General musculoskeletal complaints in a group of patients with craniomandibular disorders (cmd). A case control study. Swed Dent J 1991;15:179-85. [PubMed: 1957252]

31. Han JS, Neugebauer V. Mglur1 and mglur5 antagonists in the amygdala inhibit different components of audible and ultrasonic vocalizations in a model of arthritic pain. Pain 2005;113:211-22. [PubMed: 15621382]

32. Hollins M, Harper D, Gallagher S, Owings EW, Lim PF, Miller V, Siddiqi MQ, Maixner W. Perceived intensity and unpleasantness of cutaneous and auditory stimuli: An evaluation of the generalized hypervigilance hypothesis. Pain 2009;141:215-21. [PubMed: 19121558]

33. Hollins M, Sigurdsson A. Vibrotactile amplitude and frequency discrimination in temporomandibular disorders. Pain 1998;75:59-67. [PubMed: 9539674]

34. Hollins M, Sigurdsson A, Fillingim L, Goble AK. Vibrotactile threshold is elevated in temporomandibular disorders. Pain 1996;67:89-96. [PubMed: 8895235]

35. Jasmin L, Rabkin SD, Granato A, Boudah A, Ohara PT. Analgesia and hyperalgesia from gabamediated modulation of the cerebral cortex. Nature 2003;424:316-20. [PubMed: 12867983]

36. Jenkinson M, Bannister P, Brady M, Smith S. Improved optimization for the robust and accurate linear registration and motion correction of brain images. Neuroimage 2002;17:825-41. [PubMed: 12377157]

37. Jenkinson M, Smith S. A global optimisation method for robust affine registration of brain images. Medical Image Analysis 2001;5:143-56. [PubMed: 11516708]

38. Jensen R, Hystad T, Kvale A, Baerheim A. Quantitative sensory testing of patients with long lasting patellofemoral pain syndrome. Eur J Pain 2007;11:665-76. [PubMed: 17204440]

39. Jousmaki V, Hari R. Parchment-skin illusion: Sound-biased touch. Curr Biol 1998;8:R190. [PubMed: 9512426]

40. Kurth R, Villringer K, Mackert BM, Schwiemann J, Braun J, Curio G, Villringer A, Wolf KJ. Fmri assessment of somatotopy in human brodmann area $3 \mathrm{~b}$ by electrical finger stimulation. Neuroreport 1998;9:207-12. [PubMed: 9507956]

41. LaBar KS, Gatenby JC, Gore JC, LeDoux JE, Phelps EA. Human amygdala activation during conditioned fear acquisition and extinction: A mixed-trial fmri study. Neuron 1998;20:937-45. [PubMed: 9620698]

42. LaMotte RH, Mountcastle VB. Disorders in somesthesis following lesions of parietal lobe. J Neurophysiol 1979;42:400-19. [PubMed: 106093]

43. Lamour Y, Guilbaud G, Willer JC. Altered properties and laminar distribution of neuronal responses to peripheral stimulation in the smi cortex of the arthritic rat. Brain Res 1983;273:183-7. [PubMed: 6616226]

44. Leffler AS, Kosek E, Hansson P. The influence of pain intensity on somatosensory perception in patients suffering from subacute/chronic lateral epicondylalgia. Eur J Pain 2000;4:57-71. [PubMed: 10833556]

45. Maldjian JA, Gottschalk A, Patel RS, Detre JA, Alsop DC. The sensory somatotopic map of the human hand demonstrated at 4 tesla. Neuroimage 1999;10:55-62. [PubMed: 10385581]

46. Mao J, Mayer DJ, Price DD. Patterns of increased brain activity indicative of pain in a rat model of peripheral mononeuropathy. J Neurosci 1993;13:2689-702. [PubMed: 8388924]

47. McGlone F, Kelly EF, Trulsson M, Francis ST, Westling G, Bowtell R. Functional neuroimaging studies of human somatosensory cortex. Behav Brain Res 2002;135:147-58. [PubMed: 12356445] 
48. Melzack R. The short-form mcgill pain questionnaire. Pain 1987;30:191-7. [PubMed: 3670870]

49. Morosan P, Rademacher J, Schleicher A, Amunts K, Schormann T, Zilles K. Human primary auditory cortex: Cytoarchitectonic subdivisions and mapping into a spatial reference system. Neuroimage 2001;13:684-701. [PubMed: 11305897]

50. Moulton EA, Keaser ML, Gullapalli RP, Greenspan JD. Regional intensive and temporal patterns of functional mri activation distinguishing noxious and innocuous contact heat. J Neurophysiol 2005;93:2183-93. [PubMed: 15601733]

51. Nathan PW. Improvement in cutaneous sensibility associated with relief of pain. J Neurol Neurosurg Psychiatry 1960;23:202-6. [PubMed: 13727925]

52. Neugebauer V, Li W. Differential sensitization of amygdala neurons to afferent inputs in a model of arthritic pain. J Neurophysiol 2003;89:716-27. [PubMed: 12574449]

53. Ohara S, Crone NE, Weiss N, Treede RD, Lenz FA. Cutaneous painful laser stimuli evoke responses recorded directly from primary somatosensory cortex in awake humans. J Neurophysiol 2004;91:2734-46. [PubMed: 14602841]

54. Ozcan M, Baumgartner U, Vucurevic G, Stoeter P, Treede RD. Spatial resolution of fmri in the human parasylvian cortex: Comparison of somatosensory and auditory activation. Neuroimage 2005;25:877-87. [PubMed: 15808988]

55. Pinchoff RJ, Burkard RF, Salvi RJ, Coad ML, Lockwood AH. Modulation of tinnitus by voluntary jaw movements. Am J Otol 1998;19:785-9. [PubMed: 9831155]

56. Pleger B, Ragert P, Schwenkreis P, Forster AF, Wilimzig C, Dinse H, Nicolas V, Maier C, Tegenthoff M. Patterns of cortical reorganization parallel impaired tactile discrimination and pain intensity in complex regional pain syndrome. Neuroimage 2006;32:503-10. [PubMed: 16753306]

57. Plesh O, Wolfe F, Lane N. The relationship between fibromyalgia and temporomandibular disorders: Prevalence and symptom severity. J Rheumatol 1996;23:1948-52. [PubMed: 8923373]

58. Rollman, GB.; Gillespie, JM. Pathophysiology of pain perception. Lautenbacher, S.; Fillingim, RB., editors. Plenum; New York: 2004. p. 107-18.

59. Sawamoto N, Honda M, Okada T, Hanakawa T, Kanda M, Fukuyama H, Konishi J, Shibasaki H. Expectation of pain enhances responses to nonpainful somatosensory stimulation in the anterior cingulate cortex and parietal operculum/posterior insula: An event-related functional magnetic resonance imaging study. J Neurosci 2000;20:7438-45. [PubMed: 11007903]

60. Schurmann M, Caetano G, Hlushchuk Y, Jousmaki V, Hari R. Touch activates human auditory cortex. Neuroimage 2006;30:1325-31. [PubMed: 16488157]

61. Senapati AK, Lagraize SC, Huntington PJ, Wilson HD, Fuchs PN, Peng YB. Electrical stimulation of the anterior cingulate cortex reduces responses of rat dorsal horn neurons to mechanical stimuli. J Neurophysiol 2005;94:845-51. [PubMed: 15716373]

62. Simons SB, Tannan V, Chiu J, Favorov OV, Whitsel BL, Tommerdahl M. Amplitude-dependency of response of si cortex to flutter stimulation. BMC Neurosci 2005;6:43. [PubMed: 15969752]

63. Smith SM. Fast robust automated brain extraction. Hum Brain Mapp 2002;17:143-55. [PubMed: 12391568]

64. Smith SM, Jenkinson M, Woolrich MW, Beckmann CF, Behrens TE, Johansen-Berg H, Bannister PR, De Luca M, Drobnjak I, Flitney DE, Niazy RK, Saunders J, Vickers J, Zhang Y, De Stefano N, Brady JM, Matthews PM. Advances in functional and structural $\mathrm{mr}$ image analysis and implementation as fsl. Neuroimage 2004;23(Suppl 1):S208-19. [PubMed: 15501092]

65. Stammler T, De Col R, Seifert F, Maihofner C. Functional imaging of sensory decline and gain induced by differential noxious stimulation. Neuroimage 2008;42:1151-63. [PubMed: 18582581]

66. Tommerdahl M, Delemos KA, Favorov OV, Metz CB, Vierck CJ Jr. Whitsel BL. Response of anterior parietal cortex to different modes of same-site skin stimulation. J Neurophysiol 1998;80:3272-83. [PubMed: 9862921]

67. Tommerdahl M, Delemos KA, Vierck CJ Jr. Favorov OV, Whitsel BL. Anterior parietal cortical response to tactile and skin-heating stimuli applied to the same skin site. J Neurophysiol 1996;75:2662-70. [PubMed: 8793772]

68. Tommerdahl M, Whitsel BL, Vierck CJ Jr. Favorov O, Juliano S, Cooper B, Metz C, Nakhle B. Effects of spinal dorsal column transection on the response of monkey anterior parietal cortex to repetitive skin stimulation. Cereb Cortex 1996;6:131-55. [PubMed: 8670645] 
69. Vartiainen NV, Kirveskari E, Forss N. Central processing of tactile and nociceptive stimuli in complex regional pain syndrome. Clin Neurophysiol 2008;119:2380-8. [PubMed: 18723393]

70. Voerman VF, van Egmond J, Crul BJ. Elevated detection thresholds for mechanical stimuli in chronic pain patients: Support for a central mechanism. Arch Phys Med Rehabil 2000;81:430-5. [PubMed: 10768531]

71. Weissman DH, Roberts KC, Visscher KM, Woldorff MG. The neural bases of momentary lapses in attention. Nat Neurosci 2006;9:971-8. [PubMed: 16767087]

72. Whitsel BL, Favorov OV, Li Y, Quibrera M, Tommerdahl M. Area 3a neuron response to skin nociceptor afferent drive. Cereb Cortex 2009;19:349-66. [PubMed: 18534992]

73. Whitsel BL, Petrucelli LM, Werner G. Symmetry and connectivity in the map of the body surface in somatosensory area ii of primates. J Neurophysiol 1969;32:170-83. [PubMed: 4975532]

74. Woolrich MW, Jbabdi B, Patenaude M, Chappell S, Makni T, Behrens TE, Beckmann CF, Jenkinson M, Smith S. Bayesian analysis of neuroimaging data in fsl. Neuroimage 2009;45:S173-186. [PubMed: 19059349]

75. Yap AU, Chua EK, Dworkin SF, Tan HH, Tan KB. Multiple pains and psychosocial functioning/ psychologic distress in tmd patients. Int J Prosthodont 2002;15:461-6. [PubMed: 12375461]

76. Yau JM, Olenczak JB, Dammann JF, Bensmaia SJ. Temporal frequency channels are linked across audition and touch. Curr Biol 2009;19:561-6. [PubMed: 19268591]

77. Zhang N, Gore JC, Chen LM, Avison MJ. Dependence of bold signal change on tactile stimulus intensity in si of primates. Magn Reson Imaging 2007;25:784-94. [PubMed: 17614230]

78. Zolnoun DA, Rohl J, Moore CG, Perinetti-Liebert C, Lamvu GM, Maixner W. Overlap between orofacial pain and vulvar vestibulitis syndrome. Clin J Pain 2008;24:187-91. [PubMed: 18287822] 


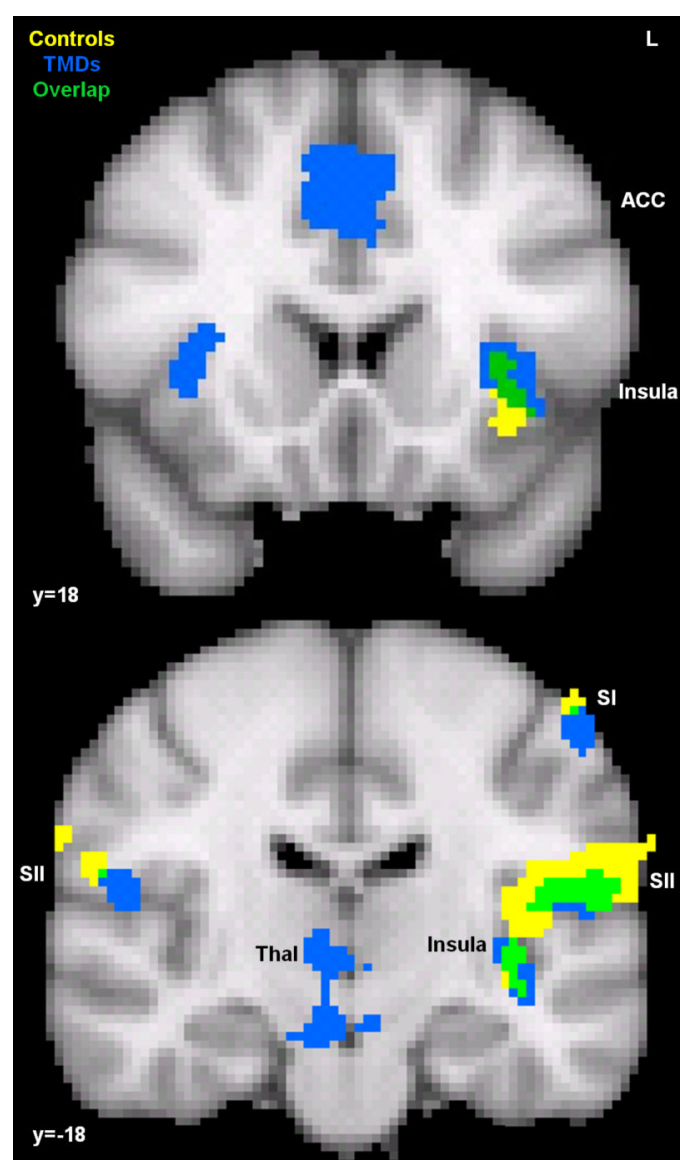

Figure 1. Active Regions

Masks of the main effect response to skin flutter for the control group only in yellow, the TMD group only in blue, and for both groups in green. A cluster mean threshold of $z>2.5$ and a cluster corrected significance of $p<0.05$ were used. Activation masks are overlayed on average anatomical images for all 25 subjects. 

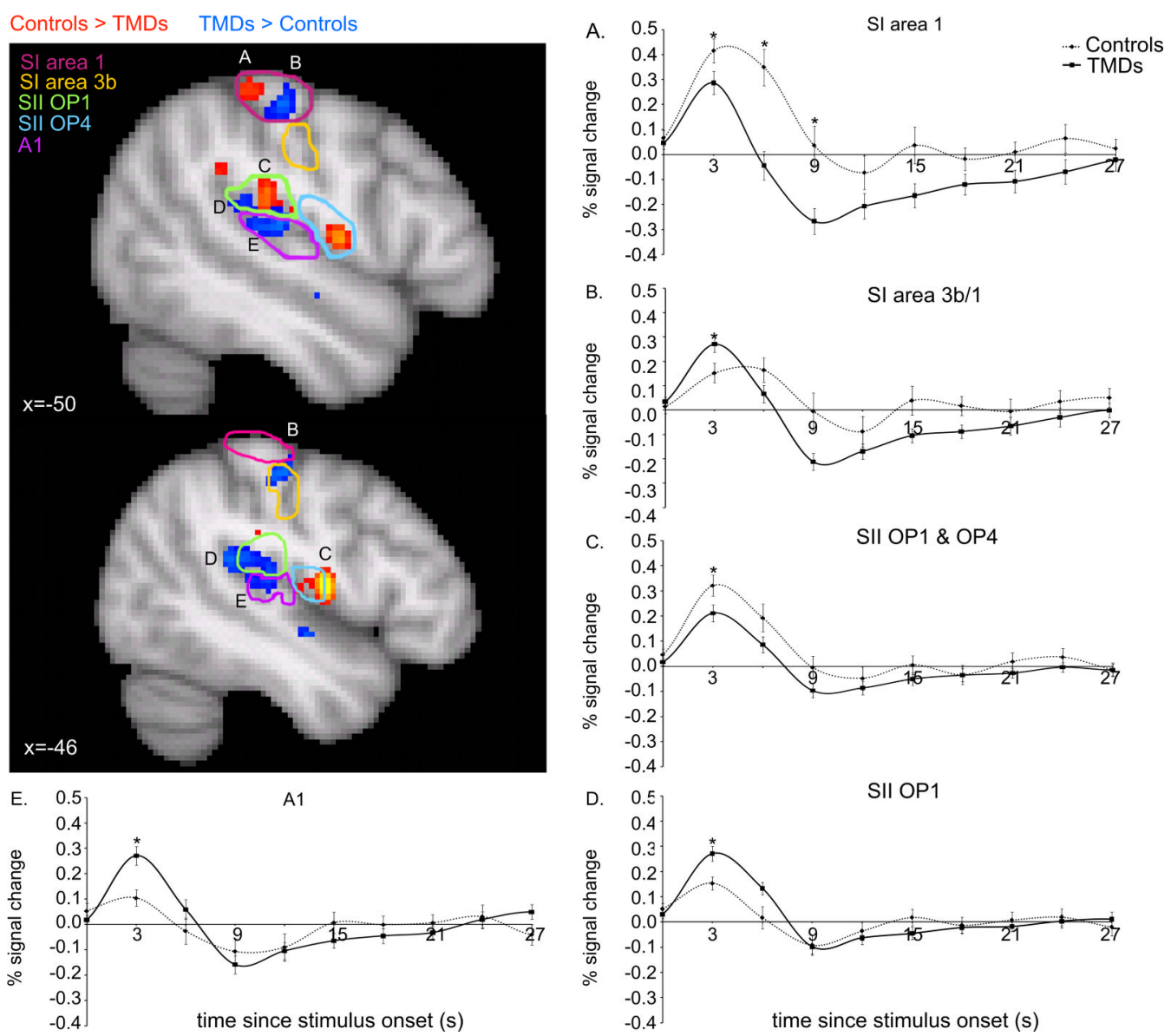

Figure 2. Comparison of mean percent signal change for controls and TMDs in subregions of somatosensory cortices contralateral to the stimulation site

(A) The subregion of SI in which controls showed greater activation than the TMD group was posterior to (B) the subregion of SI in which the peak of activation was greater for TMDs than controls. (C) \& (D) Similar dissociations in activation were found between the groups in SII with the greater evoked response in the TMD group extending to primary auditory cortex $(\mathrm{E})$. * indicates a statistically significant difference in the average percent signal change between groups at a particular time. Outlined regions are according to the Julich histological atlas. 

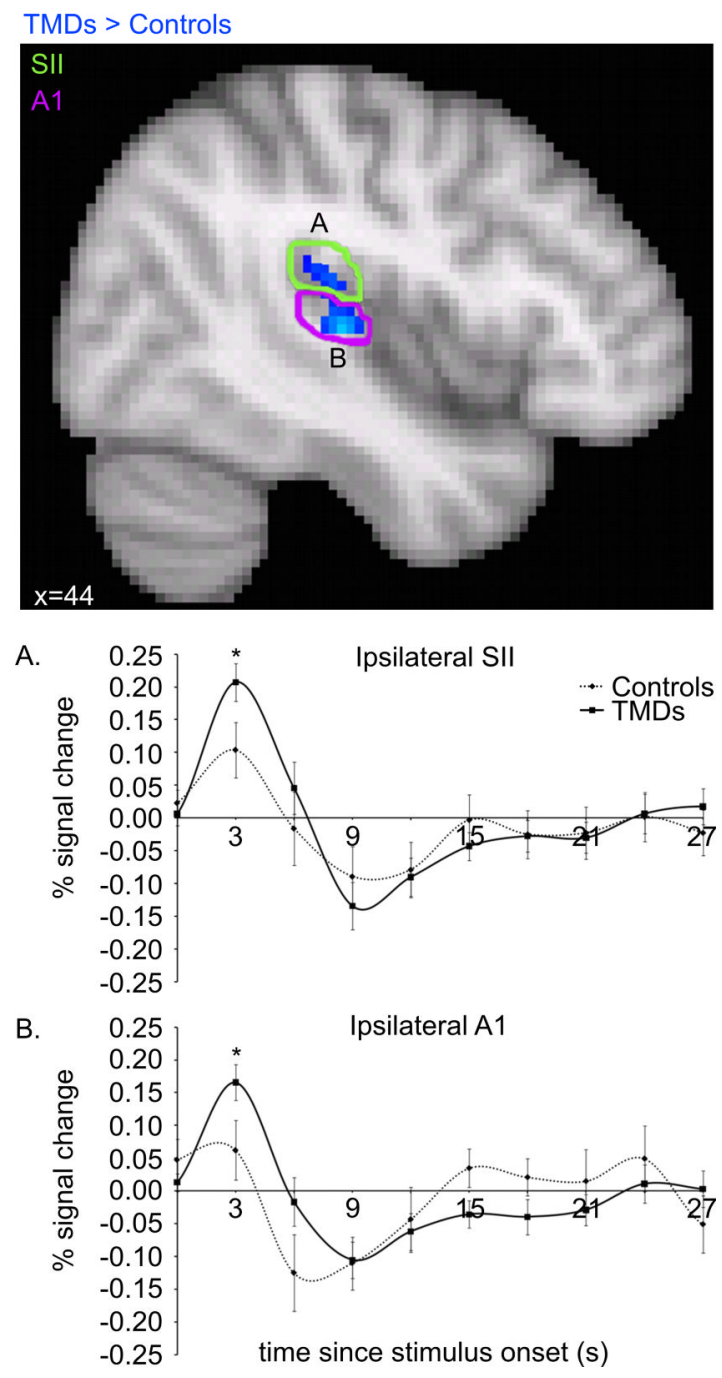

Figure 3. Comparison of mean percent signal change in SII and primary auditory cortex ipsilateral to the site of skin stimulation

The TMD group demonstrated greater activation in both (A) ipsilateral SII and (B) ipsilateral A1. Unlike on the contralateral side, there was no subregion of ipsilateral SII in which controls exhibited greater activation than TMDs in response to skin flutter. * indicates a statistically significant difference in the average percent signal change between groups at a particular time. Outlined regions are according to the Julich histological atlas. 


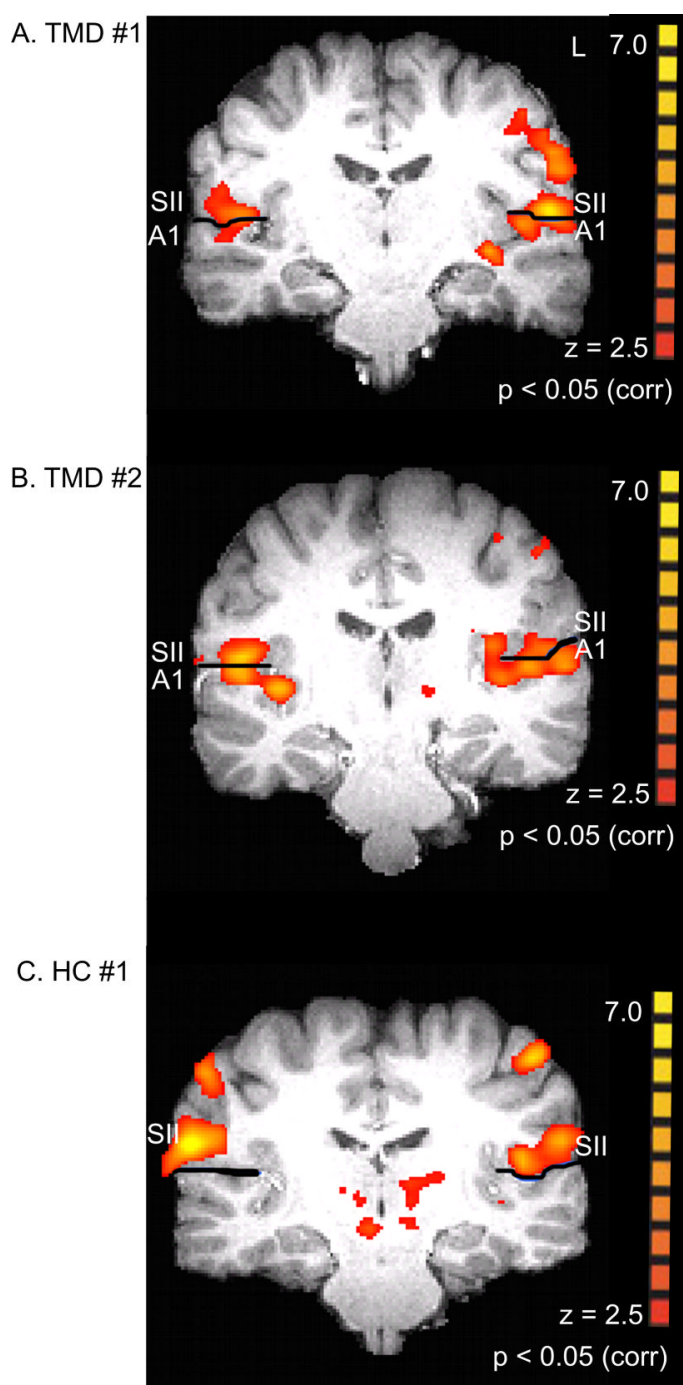

Figure 4. Representative fMRI of SII and A1 activations during finger stimulation in individual subjects

The Sylvian fissure is denoted by a black line overlaying individual activation maps. The parietal operculum (SII) is located above the Sylvian fissure while the transverse temporal gyrus (A1) is located below the Sylvian fissure. Only clusters with a mean threshold of z $>2.5$ and a cluster corrected significance of $p<0.05$ are shown. Skin flutter elicited BOLD activations on both sides of the Sylvian fissure in TMD subjects (A) and (B) but only in SII in controls $(\mathrm{C}) . \mathrm{HC}=$ Healthy Control. 


\section{TMDs $>$ Controls}

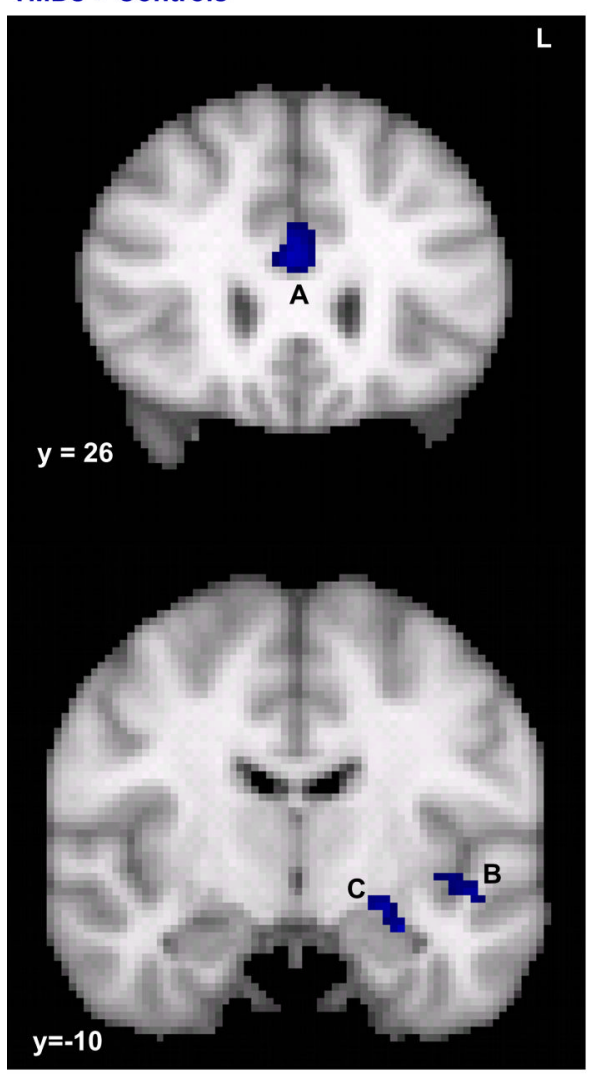

A.

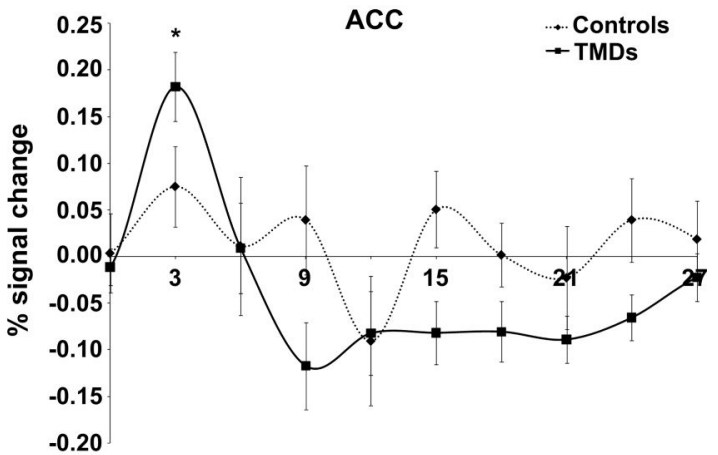

B.

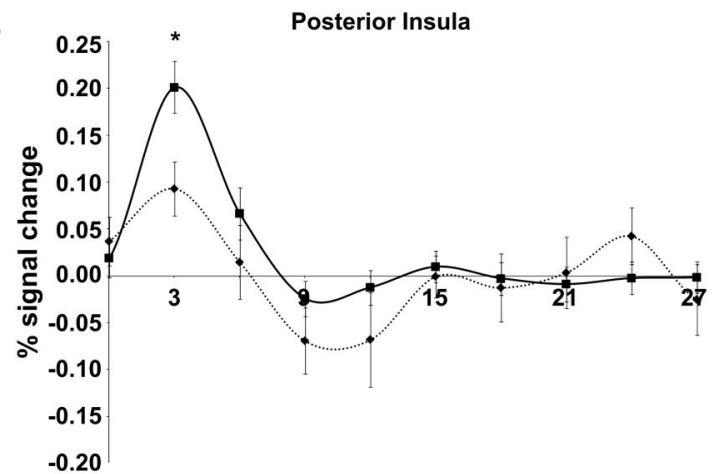

C.

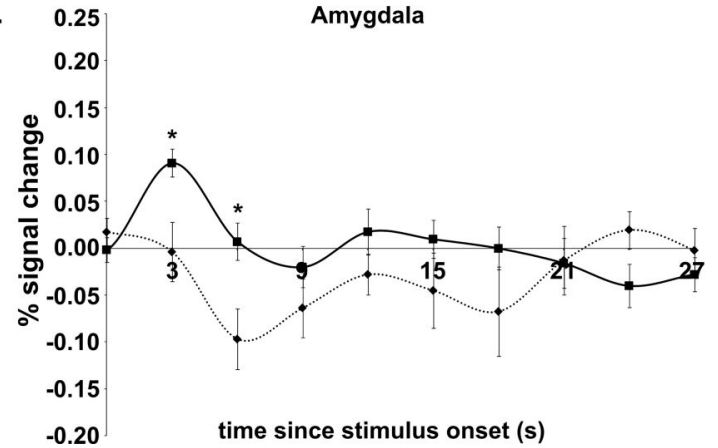

Figure 5. Comparison of mean percent signal change for controls and TMDS in (A \& B) affective and $(\mathrm{C})$ emotional processing areas

* indicates a statistically significant difference in the average percent signal change between groups at a particular time. 


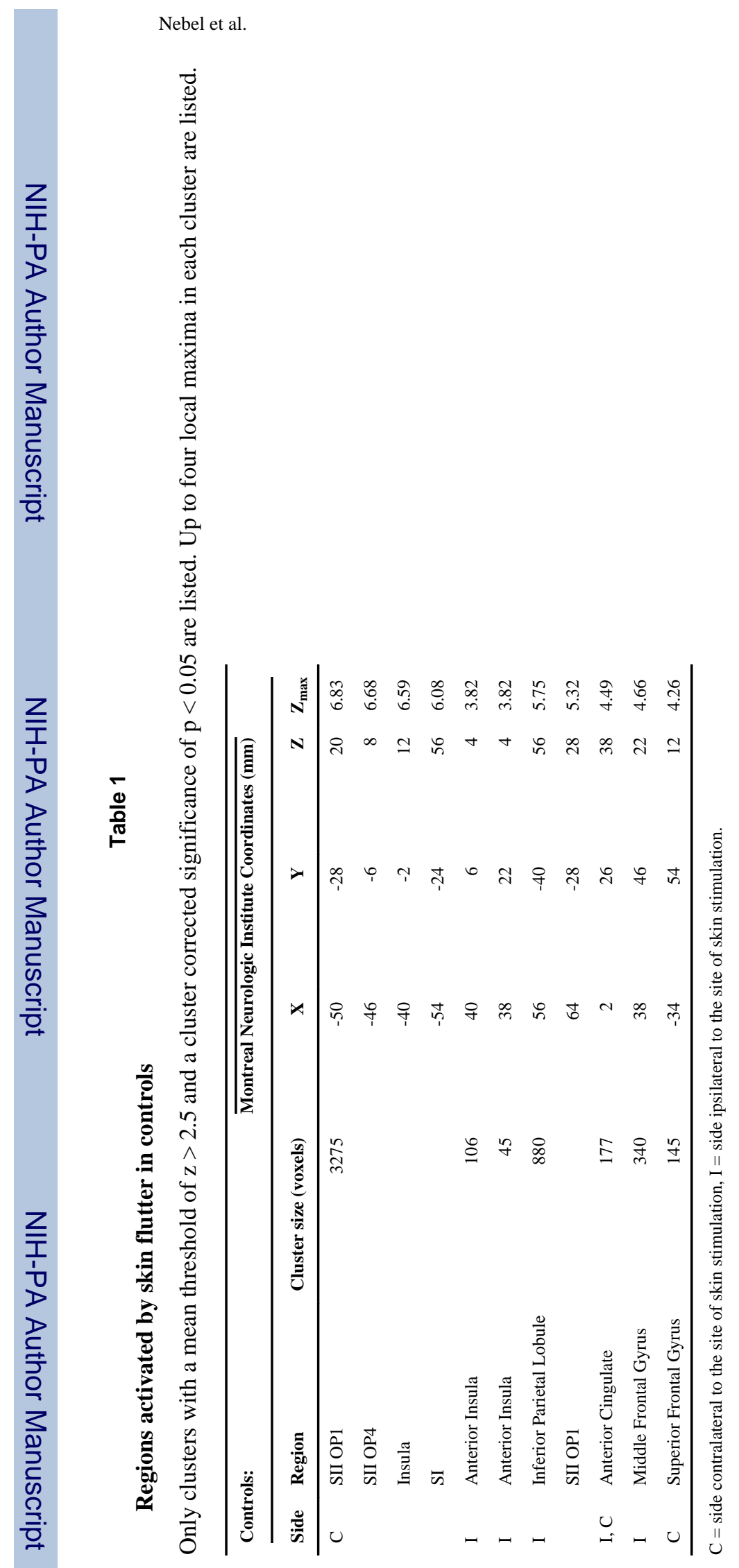

J Pain. Author manuscript; available in PMC 2011 November 1. 


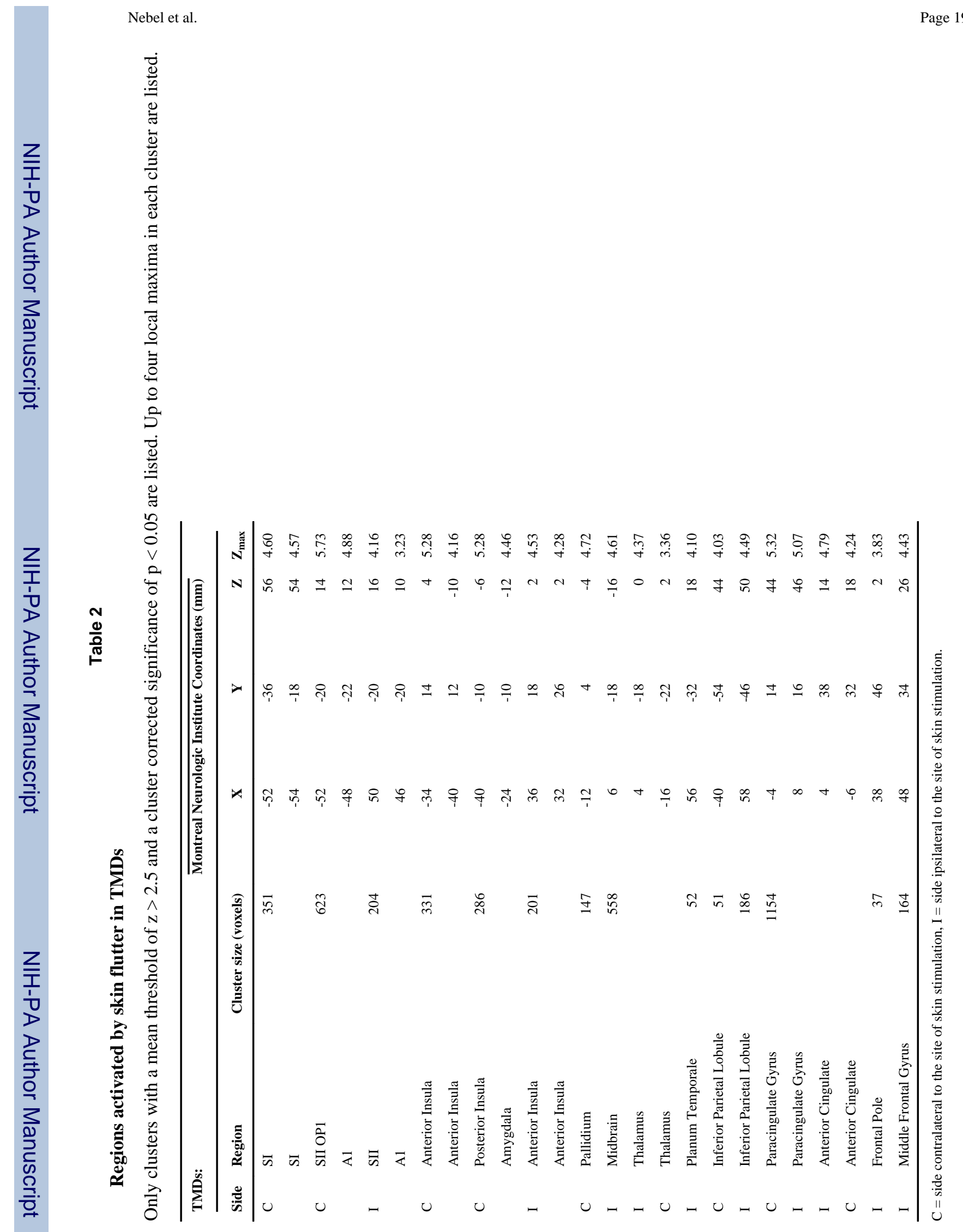

J Pain. Author manuscript; available in PMC 2011 November 1. 


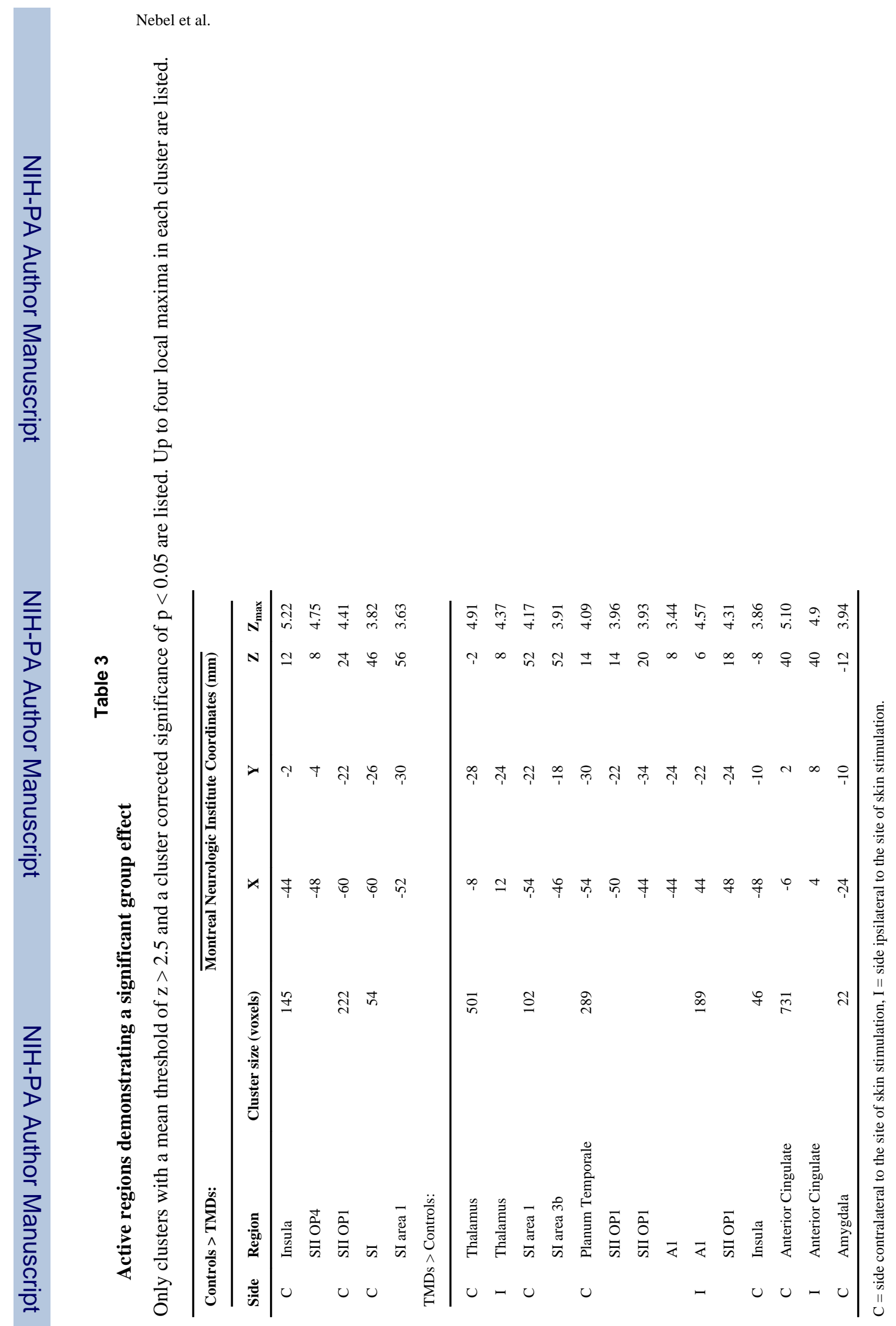

J Pain. Author manuscript; available in PMC 2011 November 1. 